\title{
Clinical Significance of has_circ_0055625 in Colon Cancer Patients Undergoing FOLFOX Chemotherapy Treatment
}

This article was published in the following Dove Press journal: International Journal of General Medicine

\section{Xiuhua Liu (D) \\ Liang Gao \\ Shaoxin Shi \\ Yun Yang}

Department of Pathology, Xiamen Haicang Hospital, Xiamen, Fujian, 361000, People's Republic of China
Correspondence: Xiuhua Liu Department of Pathology, Xiamen Haicang Hospital, 89 Haiyu Road, Haicang District, Xiamen, Fujian 361000, People's Republic of China

Tel +86-15759240618

Email liuxiuhuahc308@I63.com
Objective: Colon cancer is a leading global cancer-related cause of morbidity and mortality. The oxaliplatin, 5-fluorouracil (5-FU), and leucovorin (FOLFOX) regimen is a standard chemotherapeutic approach used to treat colon cancer. However, chemoresistant tumor cells typically lead to the emergence of recurrent FOLFOX-resistant tumors after initial treatment. As such, it is vital that novel approaches to identifying and eliminating such chemoresistant tumors be developed in an effort to improve patient chemotherapy outcomes.

Materials and Methods: In total, 100 samples of serum were obtained between April 2014 and April 2019 from patients who had been pathologically diagnosed with colon cancer from the Xiamen Haicang Hospital, and after these patients received FOLFOX chemotherapy treatment, serum samples were collected again. The expression of has_circ_0055625 in these serum samples was assessed via qPCR. Additionally, 5-FU IC50 values were detected via CCK-8 assay.

Results: We found has_circ_0055625 to be significantly upregulated in colon cancer patient serum. After FOLFOX treatment, chemotherapy-resistance was associated with the upregulation of has_circ_0055625.

Conclusion: In summary, these data may provide a foundation for future studies of chemotherapeutic resistance in patients undergoing FOLFOX treatment, potentially guiding treatment adjustment strategies. However, further work will be necessary to expand upon these findings.

Keywords: has_circ_0055625, colon cancer, chemotherapy resistance, FOLFOX chemotherapy

\section{Introduction}

Colon cancer remains one of the most prevalent and deadliest forms of cancer in the world. ${ }^{1,2}$ The oxaliplatin, 5-fluorouracil (5-FU), and leucovorin (FOLFOX) regimen is a standard chemotherapeutic regimen used to treat this cancer type. ${ }^{3-5}$ However, many patients exhibit tumor recurrence following FOLFOX treatment owing to the presence of chemoresistant tumor cells. It is thus essential that novel approaches to identifying and treating drug-resistant cancers be developed in order to improve patient outcomes.

Circular RNAs (circRNAs) are non-coding RNAs that form following the splicing of the $5^{\prime}$ and $3^{\prime}$ ends of upstream and downstream exons in an RNA molecule. ${ }^{6}$ While originally thought to be produced primarily as a consequence of inappropriate RNA splicing or gene rearrangement, there is now evidence that these 
circRNAs are closely linked to the pathology of human cancers. ${ }^{6-9}$ From a functional perspective, circRNAs can sequester microRNAs (miRNAs) in a sequence-specific manner, thereby disrupting their ability to suppress the expression of target mRNAs. ${ }^{10}$ A number of different circRNAs have recently been linked to colon cancer following identification through RNA sequencing or circRNA array assays. ${ }^{11-15}$ More work, however, is needed to understand the role of these circRNAs in the context of colon cancer chemoresistance.

Prior research suggests that has_circ_0055625 can drive colon cancer cell growth. ${ }^{14}$ As such, we chose to study the expression of this circRNA in colon cancer patients undergoing FOLFOX treatment, and we explored the relationship between the expression of has_circ_0055625, survival, and chemotherapy resistance in these patients.

\section{Materials and Methods}

\section{Patient Sample Collection}

One hundred samples of serum were obtained between April 2014 and April 2019 from patients that had been pathologically diagnosed with colon cancer from the Xiamen Haicang Hospital, and after these patients received FOLFOX chemotherapy treatment, serum samples were collected again. The Xiamen Haicang Hospital ethics committee approved the present study (No.20190321), and all patients provided written informed consent to participate. The research was performed in accordance with the Declaration of Helsinki principles.

\section{qPCR}

The expression of has_circ_0055625 in patient samples and cells was assessed via qPCR. Briefly, cDNA was generated by reverse transcribing $100 \mathrm{ng}$ of RNA per sample with a ReverTra Ace qPCR RT Kit (Toyobo Inc, Japan). All qPCR reactions were then conducted using a 7500 Real-Time PCR System (Applied Biosystems, CA, USA). The expression of target genes was calculated using the $2^{-\Delta \Delta \mathrm{Ct}}$ method: $\Delta \Delta \mathrm{Ct}=\mathrm{Ct}_{\text {has_circ_0055625 }}-\mathrm{Ct}_{\mathrm{U} 6}$. U6 small nuclear RNA was utilized as a normalization control. The has_circ_0055625 primers used for this study were as follows: forward: 5'- CCTCCT AGCCCTGTGGGCAG-3', and reverse: 5'- CCCCTGCA GGTCTGACGAGTG-3'. The U6 snRNA primers used in this study were as follows: forward: 5'CTCGCTTCGGCAGCACA-3', and reverse: 5'AACGCTTCACGAATTTGCGT-3'.

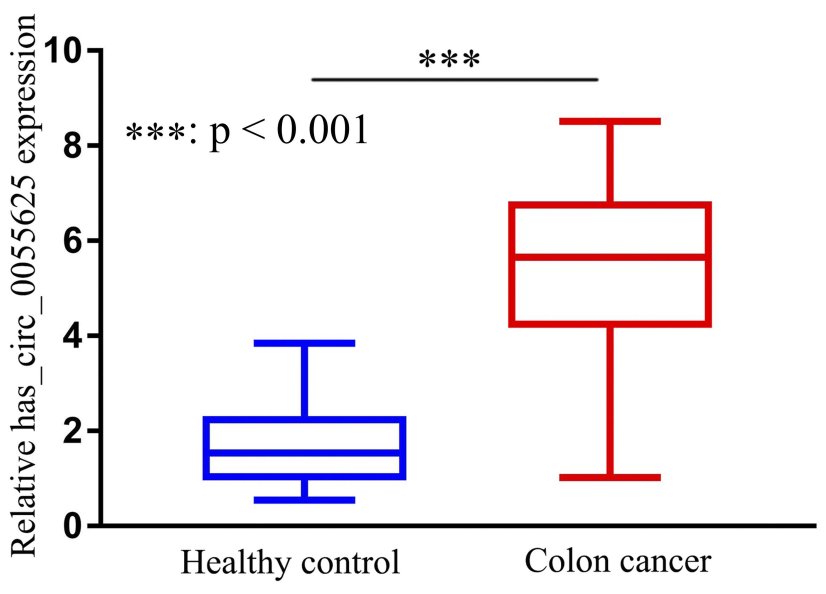

Figure I The relative expression of has_circ_0055625 in colon cancer patients prior to chemotherapy. Colon cancer patients that had not undergone FOLFOX treatment exhibited significantly higher has_circ_0055625 expression relative to healthy controls.

\section{Cell Culture and IC50 Analyses}

The SW480 and HT-29 human colon cancer cell lines and 5-FU-resistant versions thereof (SW480/5-FU and HT-29/5-FU), which were obtained by culturing these cells in the presence of 5-FU, were cultured in DMEM containing $10 \% \mathrm{FBS}$ in a $5 \% \mathrm{CO}_{2}$ incubator at $37^{\circ} \mathrm{C}$. The 5-FU IC50 values for these cells were determined with a CCK-8 kit (Vazyme Biotech, Nanjing, China).

Table I Has_circ_0055625 Expression Levels in Colon Cancer Patients

\begin{tabular}{|l|l|l|l|}
\hline Item & n (\%) & $\begin{array}{l}\text { Relative } \\
\text { has_circ_0055625 } \\
\text { Expression }\end{array}$ & $\mathbf{p}$ \\
\hline $\begin{array}{l}\text { Gender } \\
\text { Male }\end{array}$ & 48 & $5.65 \pm 1.23$ & 0.53 \\
\hline $\begin{array}{l}\text { Female (years) } \\
<55 \\
\geq 55\end{array}$ & 52 & $5.72 \pm 1.97$ & 0.35 \\
\hline $\begin{array}{l}\text { Tumor size (cm) } \\
<3\end{array}$ & 39 & $5.06 \pm 2.03$ & 0.001 \\
$\geq 3$ & $6 I$ & $5.14 \pm 2.23$ & \\
\hline $\begin{array}{l}\text { Metastasis } \\
\text { No } \\
\text { Yes }\end{array}$ & 29 & $2.15 \pm 1.06$ & $5.21 \pm 2.23$ \\
\hline $\begin{array}{l}\text { TNM stages } \\
\text { I-II } \\
\text { III-IV }\end{array}$ & 35 & $2.45 \pm 1.23$ & 0.002 \\
\hline
\end{tabular}




\section{Statistical Analysis}

SPSS 26.0 was used for all statistical testing. Data were analyzed via chi-squared tests to compare values between patients before and after treatment. Median patient survival was calculated via the Kaplan-Meier

A

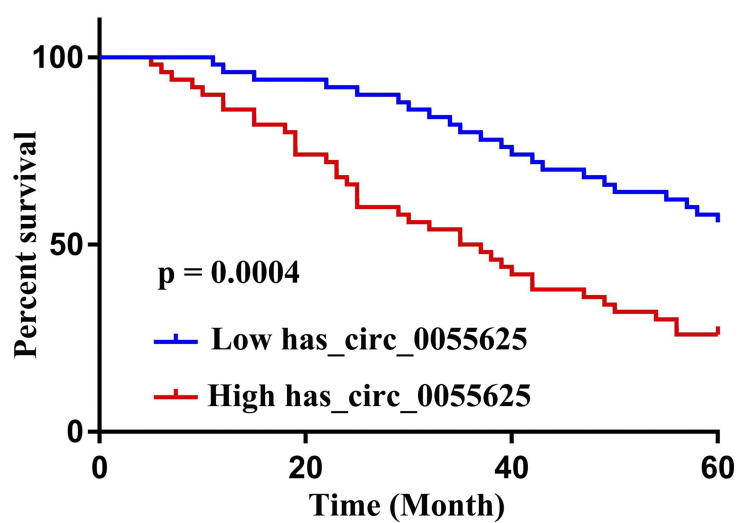

C

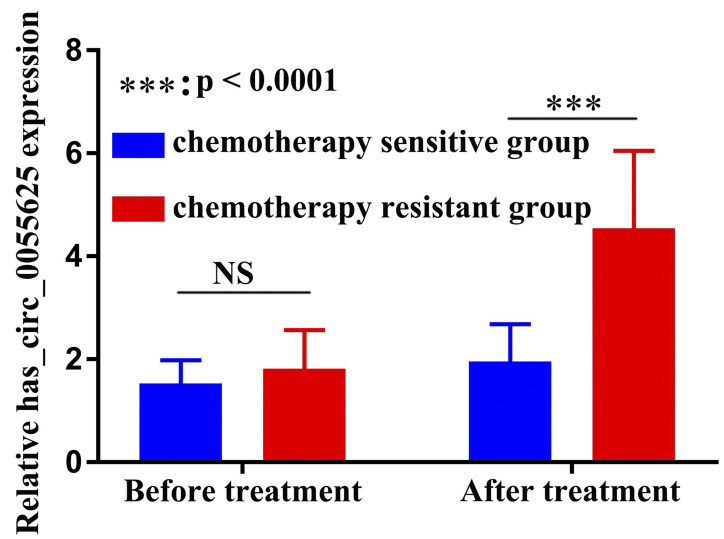

method and compared via the Log rank test. Predictors of patient survival were identified using a Cox proportional hazards model. All statistical tests were twosided, and $\mathrm{P}<0.05$ was the significance threshold for this study.

B

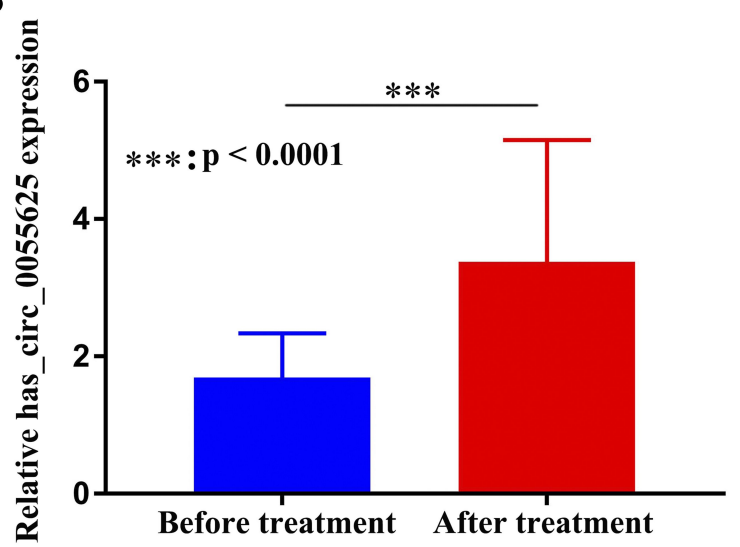

D

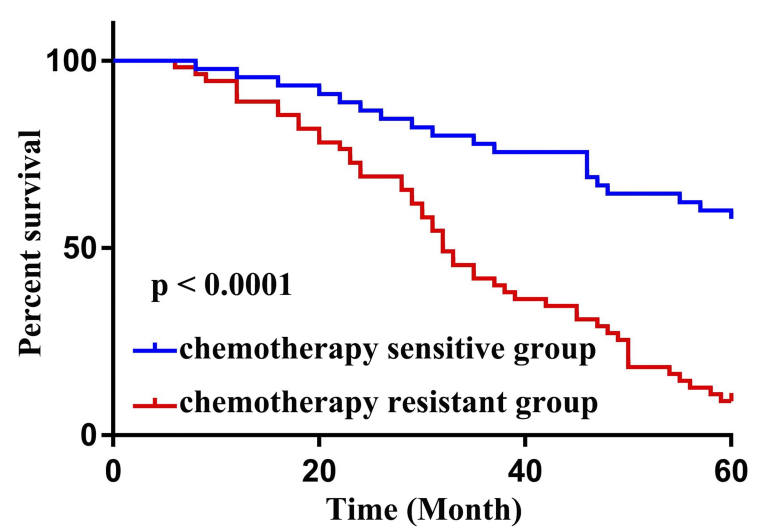

E

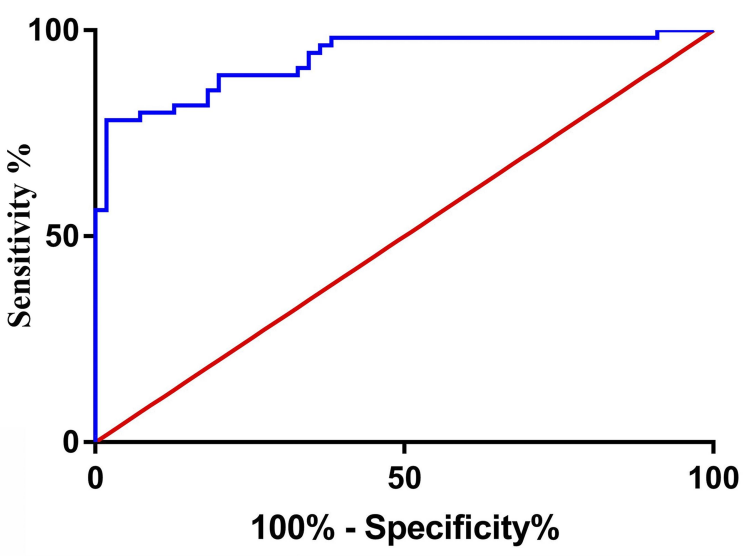

- Sensitivity\%

- Identity\%

Figure 2 The relationship between colon cancer patient survival and has_circ_0055625 expression. (A) The survival of colon cancer patients expressing high levels of has_circ_0055625 was significantly reduced relative to that of patients expressing low levels of this circRNA. (B) The expression of has_circ_0055625 in colon cancer patients was significantly elevated following FOLFOX treatment. (C) Has_circ_0055625 expression in before treatment and after treatment. (D). Chemotherapy-sensitive colon cancer patients survived for significantly longer than did chemotherapy-resistant patients. (E) Levels of has_circ_0055625 expression were found to be a potentially reliable biomarker of colon cancer patient resistance to FOLFOX chemotherapy. 
Table 2 Multiple Logistic Regression Analysis of Factors Associated with Colon Cancer Patient Survival

\begin{tabular}{|l|l|l|l|l|l|l|l|}
\hline Item & B & S.E. & Wals & Sig. & \multicolumn{1}{|l|}{ Exp (B) } & \multicolumn{2}{|c|}{ 95\% CI of Exp(B) } \\
\hline Age & 0.462 & 0.264 & 2.012 & 0.203 & 1.542 & 0.812 & 3.214 \\
Gender & 0.215 & 0.195 & 1.846 & 0.321 & 1.063 & 0.621 & 2.014 \\
High has_circ_0055625 & 3.02 & 0.63 & 21.85 & 0.000 & 16.32 & 5.06 & 41.02 \\
Stage III-IV & -1.43 & 0.532 & 6.264 & 0.008 & 0.214 & 0.08 & 0.621 \\
Metastasis & -1.624 & 0.486 & 8.862 & 0.004 & 0.322 & 0.21 & 1.023 \\
Tumor size (cm) & -1.365 & 0.456 & 6.023 & 0.007 & 0.221 & 0.365 & 0.654 \\
Constant & -0.236 & 1.210 & 0.04 & 0.764 & 0.672 & - & - \\
\hline
\end{tabular}

\section{Results}

The Expression of has_circ_0055625 in Colon Cancer Patients Prior to Chemotherapy

We found that has_circ_0055625 was expressed at significantly higher levels in samples from colon cancer patients who had not undergone chemotherapeutic treatment relative to samples from healthy controls (Figure 1). There was no apparent relationship between has_circ_0055625 expression and colon cancer patient gender or age, whereas the expression of this circRNA was associated with metastasis, tumor size, and TNM stage (Table 1).

\section{The Relationship Between Colon Cancer Patient Survival and has_circ_0055625 Expression}

We next separated 100 colon cancer patients into two groups (has_circ_0055625-low and -high) based upon median serum has_circ_0055625 expression in these patients. Subsequent Kaplan-Meier analyses revealed that patients expressing high levels of has_circ_0055625 survived for significantly less time on average relative to patients expressing low levels of this circRNA (Figure 2A). We then assessed has_circ_0055625 expression in colon cancer patients following chemotherapeutic treatment, revealing it to have been significantly upregulated following such treatment (Figure 2B). As per the Response Evaluation Criteria in Solid Tumors (RECIST) criteria, we separated FOLFOXtreated patients into chemotherapy-sensitive $(\mathrm{CR}+\mathrm{PR}$, $\mathrm{n}=45$ ) and chemotherapy-resistant ( $\mathrm{SD}+\mathrm{PD}, \mathrm{n}=55)$ groups. However, has_circ_0055625 expression levels did not vary as a function of chemotherapeutic sensitivity, while these levels did increase in chemotherapy-resistant patients following FOLFOX treatment (Figure 2C). We additionally found that chemotherapy-sensitive patients survived for significantly longer than chemotherapy-resistant patients
(Figure 2D). Importantly, we identified has_circ_0055625 expression as a potentially significant biomarker of chemotherapy resistance in colon cancer patients to FOLFOX treatment (Figure 2E).

\section{Cox Proportional Hazard Analysis}

The results of a Cox proportional hazard analysis revealed that relative has_circ_0055625 expression, tumor size, metastasis, and the clinical stage IV were all factors associated with colon cancer patient survival (Table 2), whereas survival was unrelated to patient age, gender, tumor differentiation, or pathological type.

\section{has_circ_0055625 Knockdown Reduces Colon Cancer 5-FU Resistance}

Next, we evaluated the expression of has_circ_0055625 in 5-FU-resistant colon cancer (SW480/5-FU and HT-29/5-FU) cells, revealing a significant increase in the expression of this circRNA in these resistant cell lines relative to non-resistant SW480 and HT-29 cells (Figure 3A). We then transfected SW480/5-FU and HT-29/5-FU cells with a specific siRNA construct to successfully knock down has_circ_0055625 (Figure 3B). We found that has_circ_0055625 knockdown markedly reduced the 5-FU IC50 value in SW480/5-FU/sihas_circ_0055625 and HT-29/5-FU/si-has_circ_0055625 cells relative to SW480/5-FU/si-NC and HT-29/5-FU/si-NC controls, indicating that knocking down this circRNA was sufficient to alleviate colon cancer cell decrease colon cancer cell 5-FU resistance (Figure 3C).

\section{Discussion}

Chemotherapy remains one of the most important approaches to treating patients with advanced-stage colon cancer, ${ }^{16}$ yet the emergence of chemoresistance ultimately limits the efficacy of this therapeutic strategy. ${ }^{17}$ Dysregulated circRNA expression within cancer cells can alter the ability of these non-coding RNAs to regulate 
A
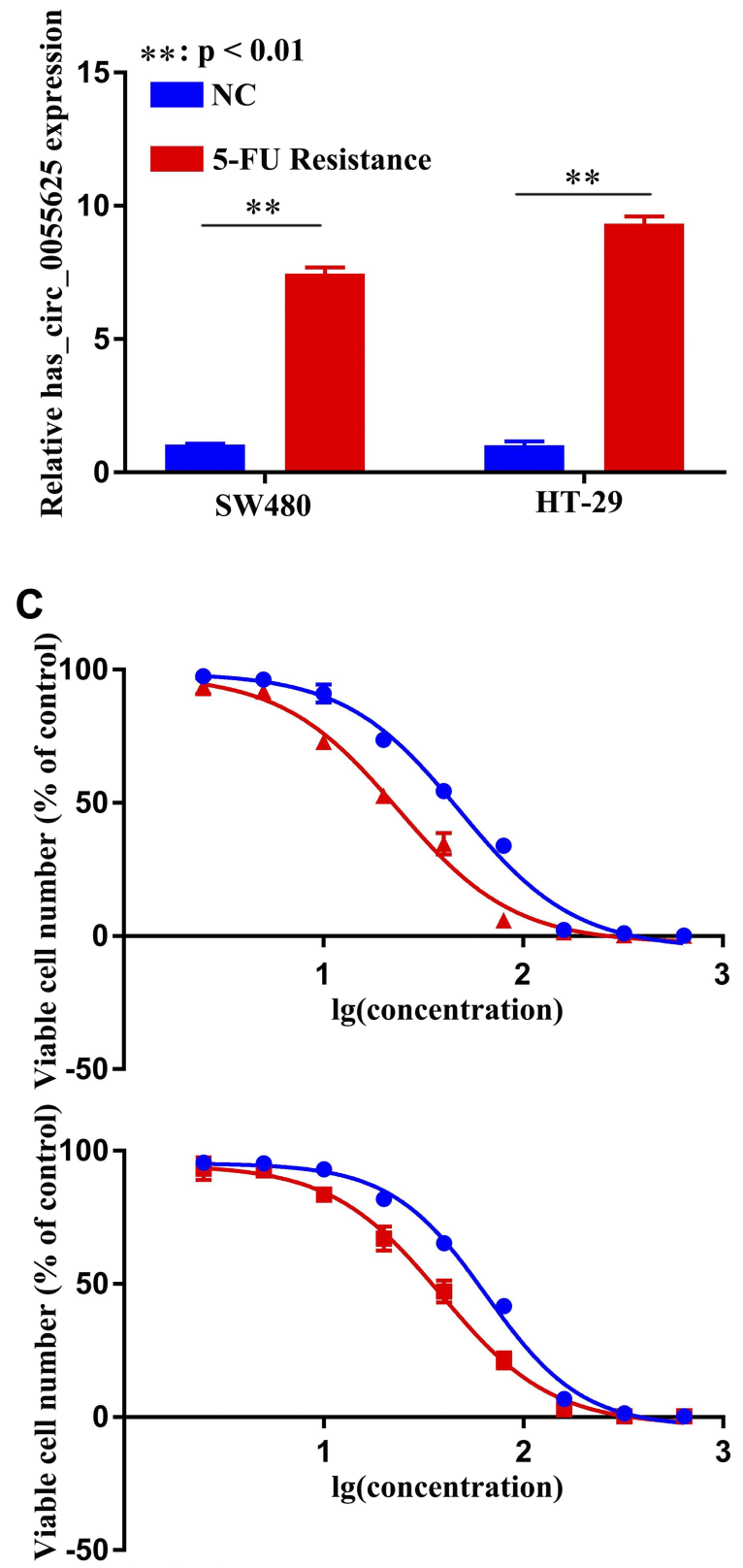

B

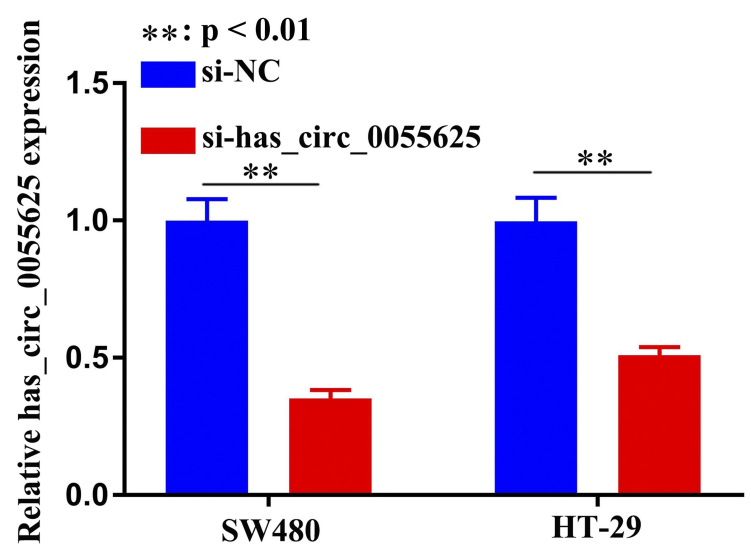

Figure 3 Knocking down has_circ_0055625 decreases colon cancer cell line resistance to 5-FU. (A). The expression of has_circ_0055625was analyzed in SW480/5-FU or HT-29/5-FU cells. (B). The expression of has_circ_0055625 was analyzed in SW480/5-FU/si-has_circ_0055625 and HT-29/5-FU/si-has_circ_0055625 cells. (C). 5-FU IC50 values were determined via CCK-8 assay.

miRNA activity and protein expression. ${ }^{18-20}$ When these circRNAs influence miRNAs and proteins responsible for controlling drug metabolism, absorption, or receptor expression, they may markedly alter the efficacy of chemotherapeutic treatments if dysregulated. For example, circRNA_101237 is a prognostic biomarker associated with resistance to cisplatin in hepatocellular carcinoma. ${ }^{21}$ In addition, circFBXO11 regulates the progression and oxaliplatin resistance of hepatocellular carcinoma by modulating the miR-605/FOXO3/ABCB1 axis, ${ }^{22}$ whereas hsa_circ_0079662 induces human colon cancer cell resistance to oxaliplatin via affecting the TNF- $\alpha$ pathway. ${ }^{23}$ Sequencing studies have also found that mRNA, circRNA, and IncRNA expression profiles in extracellular vesicles derived from human serum samples offer promise as diagnostic cancer biomarkers. ${ }^{24}$ Measuring serum circRNA levels may thus represent a therapeutically viable approach to predicting patient treatment and survival outcomes. 
Prior research has demonstrated that has_circ_0055625 is dysregulated in colon cancer [14]. Our present results further confirmed that this circRNA was upregulated in the serum of colon cancer patients, and this upregulation was correlated with patient clinical staging and overall prognosis. Specifically, patients expressing high levels of high has_circ_0055625 survived for significantly less time than did patients expressing low levels of this circRNA. While there were no significant differences in the expression of has_circ_0055625 when comparing patients who were sensitive and resistant to FOLFOX treatment, we did find that such treatment was associated with a significant increase in serum has_circ_0055625 levels relative to pretreatment expression thereof. Chemotherapy-sensitive colon cancer patients also survived for significantly longer than did chemotherapyresistant patients, and has_circ_0055625 expression level was identified as a potentially significant biomarker of FOLFOX resistance in these colon cancer patients. We further determined that knocking down has_circ_0055625 was sufficient to alleviate colon cancer cell line resistance to 5-FU. As such, we believe that further in-depth analyses of the therapeutic and diagnostic relevance of this and other serum circRNAs may help to guide appropriate colon cancer patient treatment.

In summary, we found that has_circ_0055625 was upregulated in colon cancer patient serum, and that FOLFOX chemotherapeutic treatment further upregulated this circRNA in chemotherapy-resistant patients. Relative to chemotherapy-resistant patients, has_circ_0055625 was more significantly downregulated in chemotherapysensitive patients, suggesting that this circRNA represents a promising research target for the study of mechanisms of FOLFOX resistance. However, further research will be required to validate and expand upon our findings.

\section{Acknowledgments}

The work was supported by the Social development and soft science project of Haicang District, Xiamen (No. 350205Z20184003)

\section{Disclosure}

The authors report no conflicts of interest for this work.

\section{References}

1. Siegel RL, Miller KD, Jemal A. Cancer statistics, 2020. CA Cancer J Clin. 2020;70(1):7-30. doi:10.3322/caac.21590
2. Siegel RL, Miller KD, Goding Sauer A, et al. Colorectal cancer statistics, 2020. CA Cancer J Clin. 2020;70(3):145-164. doi:10.3322/caac. 21601

3. Muneoka K, Shirai Y, Sasaki M, Wakai T, Sakata J, Hatakeyama K. Interstitial pneumonia arising in a patient treated with oxaliplatin, 5-fluorouracil, and, leucovorin (FOLFOX). Int J Clin Oncol. 2009;14 (5):457-459. doi:10.1007/s10147-008-0863-2

4. Aballea S, Chancellor JV, Raikou M, et al. Cost-effectiveness analysis of oxaliplatin compared with 5-fluorouracil/leucovorin in adjuvant treatment of stage III colon cancer in the US. Cancer. 2007;109 (6):1082-1089. doi:10.1002/cncr.22512

5. Lenz HJ, Van Cutsem E, Khambata-Ford S, et al. Multicenter Phase II and translational study of cetuximab in metastatic colorectal carcinoma refractory to irinotecan, oxaliplatin, and fluoropyrimidines. $J \quad$ Clin Oncol. 2006;24(30):4914-4921. doi:10.1200/ JCO.2006.06.7595

6. Zhang Y, Liang W, Zhang P, et al. Circular RNAs: emerging cancer biomarkers and targets. J Exp Clin Cancer Res. 2017;36(1):152. doi:10.1186/s13046-017-0624-z

7. Meng S, Zhou H, Feng Z, et al. CircRNA: functions and properties of a novel potential biomarker for cancer. Mol Cancer. 2017;16(1):94. doi:10.1186/s12943-017-0663-2

8. Greene J, Baird AM, Brady L, et al. Circular RNAs: biogenesis, Function and Role in Human Diseases. Front Mol Biosci. 2017;4:38. doi:10.3389/fmolb.2017.00038

9. Han YN, Xia SQ, Zhang YY, Zheng JH, Circular LW. RNAs: A novel type of biomarker and genetic tools in cancer. Oncotarget. 2017;8(38):64551-64563. doi:10.18632/oncotarget.18350

10. Rong D, Sun H, Li Z, et al. An emerging function of circRNA-miRNAs-mRNA axis in human diseases. Oncotarget. 2017;8(42):73271-73281. doi:10.18632/oncotarget.19154

11. Min L, Wang H, Zeng Y. CircRNA_104916 regulates migration, apoptosis and epithelial-mesenchymal transition in colon cancer cells. Front Biosci. 2019;24(5):819-832. doi:10.2741/4753

12. Hsiao KY, Lin YC, Gupta SK, et al. Noncoding Effects of Circular RNA CCDC66 Promote Colon Cancer Growth and Metastasis. Cancer Res. 2017;77(9):2339-2350. doi:10.1158/0008-5472.CAN16-1883

13. Zhou P, Xie W, Huang HL, et al. circRNA 100859 functions as an oncogene in colon cancer by sponging the miR-217-HIF-1alpha pathway. Aging. 2020;12(13):13338-13353. doi:10.18632/ aging. 103438

14. Zhang J, Liu H, Zhao P, Zhou H, Mao T. Has_circ_0055625 from circRNA profile increases colon cancer cell growth by sponging miR-106b-5p. J Cell Biochem. 2019;120(3):3027-3037. doi: $10.1002 /$ jcb. 27355

15. He JH, Li YG, Han ZP, et al. The CircRNA-ACAP2/Hsa-miR-21-5p/ Tiam1 Regulatory Feedback Circuit Affects the Proliferation, Migration, and Invasion of Colon Cancer SW480 Cells. Cell Physiol Biochem. 2018;49(4):1539-1550. doi:10.1159/000493457

16. Arredondo J, Baixauli J, Pastor C, et al. Mid-term oncologic outcome of a novel approach for locally advanced colon cancer with neoadjuvant chemotherapy and surgery. Clin Transl Oncol. 2017;19 (3):379-385. doi:10.1007/s12094-016-1539-4

17. Chikazawa N, Tanaka H, Tasaka T, et al. Inhibition of Wnt signaling pathway decreases chemotherapy-resistant side-population colon cancer cells. Anticancer Res. 2010;30(6):2041-2048.

18. Shan G, Shao B, Liu Q, et al. circFMN2 Sponges miR-1238 to Promote the Expression of LIM-Homeobox Gene 2 in Prostate Cancer Cells. Mol Ther Nucleic Acids. 2020;21:133-146. doi:10.1016/j.omtn.2020.05.008

19. Li Y, Li C, Xu R, Wang Y, Li D, Zhang B. A novel circFMN2 promotes tumor proliferation in CRC by regulating the miR-1182/ hTERT signaling pathways. Clin Sci (Lond). 2019;133 (24):2463-2479. doi:10.1042/CS20190715 
20. Guan E, Xu X, Xue F. circ-NOTCH1 acts as a sponge of miR-637 and affects the expression of its target gene Apelin to regulate gastric cancer cell growth. Biochem Cell Biol. 2020;98(2):164-170. doi:10.1139/bcb-2019-0079

21. Zhou S, Wei J, Wang Y, Liu X. Cisplatin resistance-associated circRNA_101237 serves as a prognostic biomarker in hepatocellular carcinoma. Exp Ther Med. 2020;19(4):2733-2740. doi:10.3892/ etm. 2020.8526

22. Li J, Qin X, Wu R, Wan L, Zhang L, Circular LR. RNA circFBXO11 modulates hepatocellular carcinoma progress and oxaliplatin resistance through miR-605/FOXO3/ABCB1 axis. $J$ Cell Mol Med. 2020;24(9):5152-5161. doi:10.1111/jcmm.15162
23. Lai M, Liu G, Li R, et al. Hsa circ 0079662 induces the resistance mechanism of the chemotherapy drug oxaliplatin through the TNF-alpha pathway in human colon cancer. J Cell Mol Med. 2020;24(9):5021-5027. doi:10.1111/jcmm.15122

24. Li Y, Zhao J, Yu S, et al. Extracellular Vesicles Long RNA Sequencing Reveals Abundant mRNA, circRNA, and lncRNA in Human Blood as Potential Biomarkers for Cancer Diagnosis. Clin Chem. 2019;65(6):798-808. doi:10.1373/clinchem.2018.301291

\section{Publish your work in this journal}

The International Journal of General Medicine is an international, peer-reviewed open-access journal that focuses on general and internal medicine, pathogenesis, epidemiology, diagnosis, monitoring and treatment protocols. The journal is characterized by the rapid reporting of reviews, original research and clinical studies across all disease areas. The manuscript management system is completely online and includes a very quick and fair peer-review system, which is all easy to use. Visit http://www.dovepress.com/ testimonials.php to read real quotes from published authors.

Submit your manuscript here: https://www.dovepress.com/international-journal-of-general-medicine-journal 